\title{
X-ray scattering in the vorticity direction and rheometry from confined fluids
}

\author{
Patrick Pfleiderer, ${ }^{1,2}$ Seung Jae Baik, ${ }^{1}$ Zhenkun Zhang, ${ }^{1,3}$ Giovanni Vleminckx, ${ }^{1}$ \\ Minne Paul Lettinga, ${ }^{4,5}$ Eric Grelet, ${ }^{6}$ Jan Vermant, ${ }^{1,7}$ and Christian Clasen ${ }^{1}$ \\ ${ }^{1}$ Department of Chemical Engineering, KU Leuven, 3001 Leuven, Belgium \\ ${ }^{2}$ Department of Physics, University of Konstanz, 78457 Konstanz, Germany \\ ${ }^{3}$ College of Chemistry, Nankai University, Tianjin 300071, China \\ ${ }^{4}$ Institute of Complex Systems (ICS-3), Forschungszentrum Jülich, 52425 Jülich, Germany \\ ${ }^{5}$ Department for Physics and Astronomy, KU Leuven, 3001 Leuven, Belgium \\ ${ }^{6}$ Centre de Recherche Paul-Pascal, CNRS - Université de Bordeaux, 33600 Pessac, France \\ ${ }^{7}$ Department of Materials, ETH Zürich, 8093 Zürich, Switzerland
}

(Received 3 March 2014; accepted 25 May 2014; published online 13 June 2014)

\begin{abstract}
An X-ray flexure-based microgap rheometer (X-FMR) has been designed for combining rheology and in situ small-angle X-ray scattering from the vorticity plane. The gap distance can be varied continuously from $500 \mu \mathrm{m}$ down to several $\mu \mathrm{m}$, which provides the unique possibility to generate a strong confinement for many complex fluids. A singular advantage of this setup is the possibility to directly probe the vorticity direction of the flow field with a microfocus X-ray beam and to probe the structural response of the fluid to combined shear and confinement in the vorticity plane. The sliding-plate setup operates over a wide range of shear rates of $\dot{\gamma}=10^{-3}-10^{3} \mathrm{~s}^{-1}$ and strains in the range of $10^{-4}-10^{2}$. The flexure-based bearing maintains the plate parallelism within $10^{-5}$ rad. The X-FMR requires very small sample volumes on the order of $10 \mu 1$. The applicability of the device is demonstrated here with limited examples of a nematic suspension of $f d$ virus (rods), and a crystalline suspension containing sterically stabilized polystyrene-butylacrylate latex particles. (C) 2014 AIP Publishing LLC. [http://dx.doi.org/10.1063/1.4881796]
\end{abstract}

\section{INTRODUCTION}

Studies that combine small-angle scattering and rheology have been extremely useful for elucidating the flow-induced structure of a variety of complex fluids under flow. X-rays in particular can be used to study the structure, both in situ and time-resolved, of a wide range of material classes. Hence, they have been applied to both stable and flocculated colloidal suspensions, ${ }^{1-5}$ surfactant systems,${ }^{6-8}$ liquid crystalline polymers, ${ }^{9-12}$ flow enhanced crystallization in polymers, ${ }^{13-17}$ and polymer nanocomposites. ${ }^{18,19}$ The advent of highly collimated beams and intense X-ray sources, both using synchrotron radiation but also with modern lab sources, has made small angle (SAXS) and even ultra-small angle X-ray scattering (USAXS) techniques available which can be used to explore a wide range of length scales ${ }^{5}\left(\mathbf{q}=0.001-1 \mathrm{~nm}^{-1}\right)$ with an excellent resolving power, during flow and with very good time resolution. This makes it ideally suited to help elucidate the link between the non-linear rheological (mechanical) response of materials with the underlying changes in the microstructure. X-ray scattering allows one to probe either effects of the shape, orientation, and size of individual objects, as characterized by the form factor $P(\mathbf{q})$, or effects of the spatial organization of objects, which is characterized by the structure factor $S(\mathbf{q})$ in reciprocal space.

In the small or ultra small angle limit, a two-dimensional detector probes scattering vectors, $\mathbf{q}$, that reside in a single plane of the flow field. Depending on the nature of the flow cell and the relative orientation of the orientation of the incident X-ray beam to the flow cell different components of the structure and form factors can be probed in that plane. Couette geometries have been most commonly used, first as standalone shearing devices ${ }^{20,21}$ and more recently for combined rheology and scattering measurements. ${ }^{4,22}$ When the Xray beam is sent along the centerline of the Couette cell, the form and structure factor are projected in the $\mathbf{v}-\boldsymbol{\omega}$ plane (see Fig. 1). For incident radiation travelling along the flow direction, $P(\mathbf{q})$ and $S(\mathbf{q})$ are projected into the $\boldsymbol{\omega}-\nabla \mathbf{v}$ plane. However, measurements of $P(\mathbf{q})$ and $S(\mathbf{q})$ projected in the $\mathbf{v}-\nabla \mathbf{v}$ plane are most relevant for understanding the link with the shear and time-dependent viscosity, yet they are the most difficult to perform.

Different approaches for probing the flow-induced structural changes in the $\mathbf{v}-\nabla \mathbf{v}$ plane have been proposed. An obvious approach is to sent the radiation along the vorticity direction of the flow, as has been done for light scattering methods. ${ }^{23}$ However, flipping the direction of the X-ray beam around is not that easy and requires a dedicated detection line. ${ }^{24-27}$ An alternate solution, used in neutron scattering experiments by Wagner and co-workers, ${ }^{28,29}$ is to close the top of the Couette cell and turn it by $90^{\circ}$. However, the path length for the radiation to traverse is significant and absorption or multiple scattering effects impose limits on the applicability of this technique for SAXS studies. Careful design of the flow cell is also required to avoid the effects of 3D flow or instabilities such as shear banding in a relatively wide gap Couette. ${ }^{28,30}$ The most successful approach in investigating the structure in the $\mathbf{v}-\nabla \mathbf{v}$ plane using X-ray scattering has been obtained by Burghardt and co-workers using an annular cone device. ${ }^{31,32}$ Their setup uses a design first proposed by 


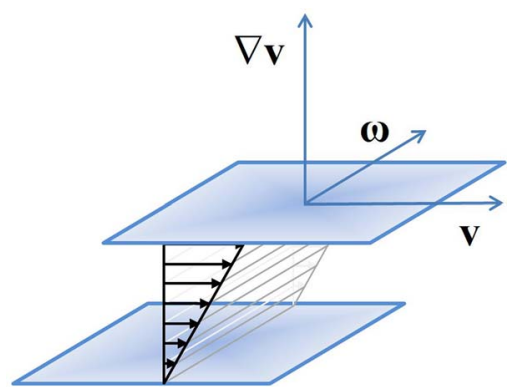

FIG. 1. Schematic drawing of a plane Couette shear flow field indicating the three primary directions: flow $(\mathbf{v})$, velocity gradient $(\nabla \mathbf{v})$, and vorticity $(\omega)$ direction.

Wales and Janeschitz-Krieg ${ }^{33}$ for rheo-optical experiments, consisting of an annular ring device in which the beam is sent horizontally across the gap of an annular cone or plateplate. Similar setups have successfully been used for Neutron scattering. ${ }^{34}$ In the case of X-ray scattering, spatial and high temporal resolution can be obtained. ${ }^{19,35,36}$ However, due to the nature of the flow geometry it is difficult to obtain accurate rheological measurements, and changing the gap size is also not very easy because of the requirement for the beam to traverse an inclined geometry.

In the present work, we propose the use of a sliding plate rheometer with a specially designed geometry for studying the structure in the vorticity or $\mathbf{v}-\nabla \mathbf{v}$ plane. The instrument is based on the general concept of sliding plate rheometry ${ }^{37-39}$ and its extension to the flexure based microgap rheometer (FMR) developed by McKinley and co-workers. ${ }^{40-42}$ The excellent alignment and parallelism of the FMR also enable one to study confinement effects (when the length scales of the fluid and the gap interfere), ${ }^{43}$ absolute slip, ${ }^{44,45}$ and the investigation of $\mu 1$ amounts of fluid samples. ${ }^{46-48}$ The flow field in a sliding plate setup is homogeneous and rheometric. This offers an advantage over other approaches to study confinement effects, such as microfluidic techniques where the flow fields are complex and non-homogeneous, making it difficult to analyze the complex material response in a flow field where strain and flow history vary.

In the following, we present in detail the design and operational space of a small, portable sliding plate rheometer based on the FMR for use in an X-ray setup. The unique applicability of this X-ray flexure-based microgap rheometer (X-FMR) for in situ SAXS measurements in the vorticity direction under confined flow conditions is demonstrated with case studies of sterically stabilized latex dispersions and suspension of semi-rigid model virus particles (rods).

\section{X-FMR SETUP}

\section{A. Aluminum frame with compound flexure systems}

Figs. 2 and 3 show a schematic drawing and an image of the X-FMR. The main structural component is a monolithic

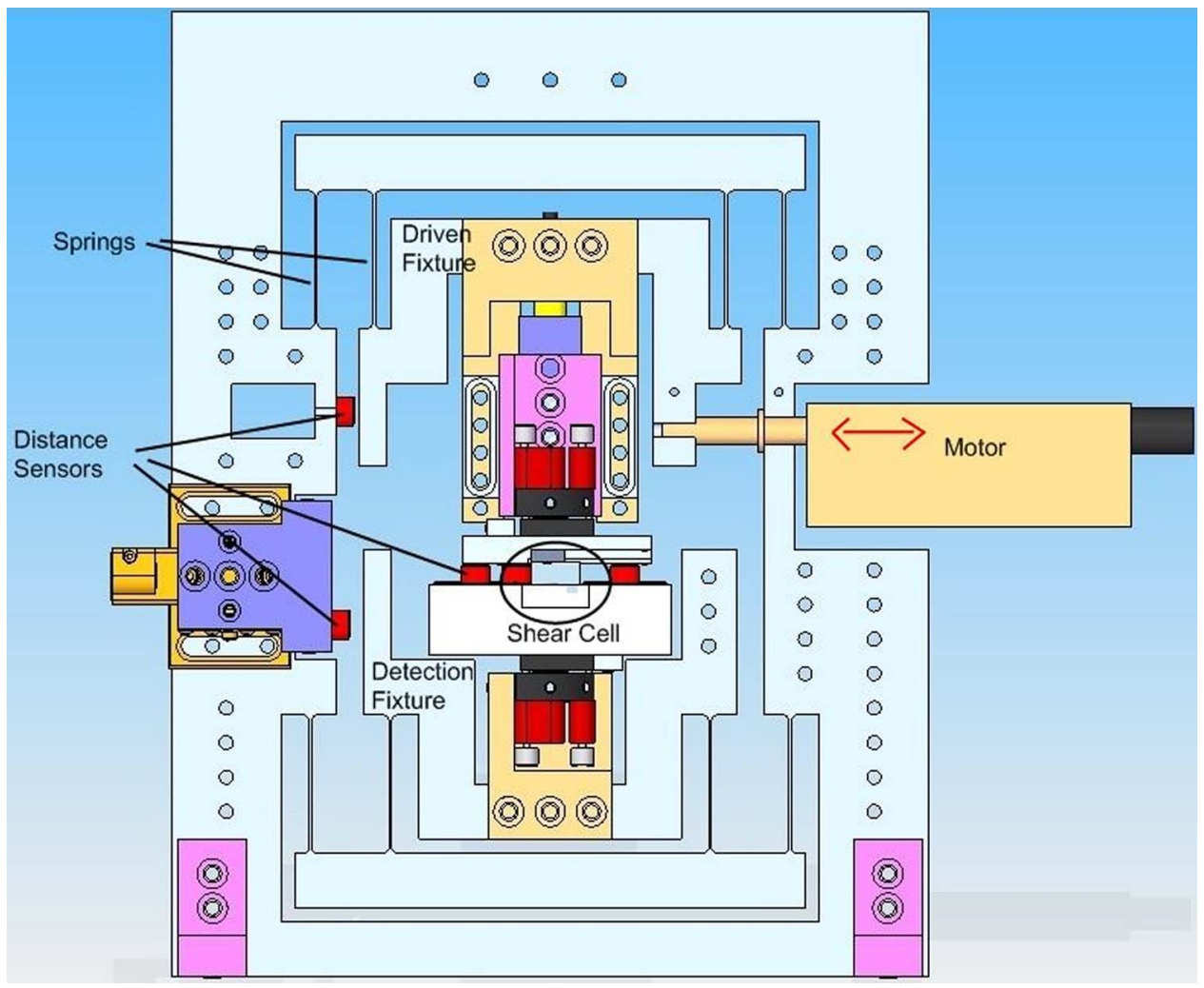

FIG. 2. CAD drawing of the X-FMR setup. The monolithic aluminum frame (frame width for scale: $23.5 \mathrm{~cm}$ ) incorporates thin (leaf) springs that carry via the upper (driven) and the lower (detecting) fixture the upper and lower surfaces of the shear cell. The geometric arrangement of the springs ensures precisely parallel motion of upper and lower fixtures. The upper (driven) fixture is displaced by a motor (red arrows). The small displacement of the lower (detection) fixture is proportional to the stress arising from the sheared sample. Non-contact distance sensors monitor the displacement of both fixtures. A vertical translation stage within the upper fixture sets the gap distance; it can be manually lifted for sample loading. 


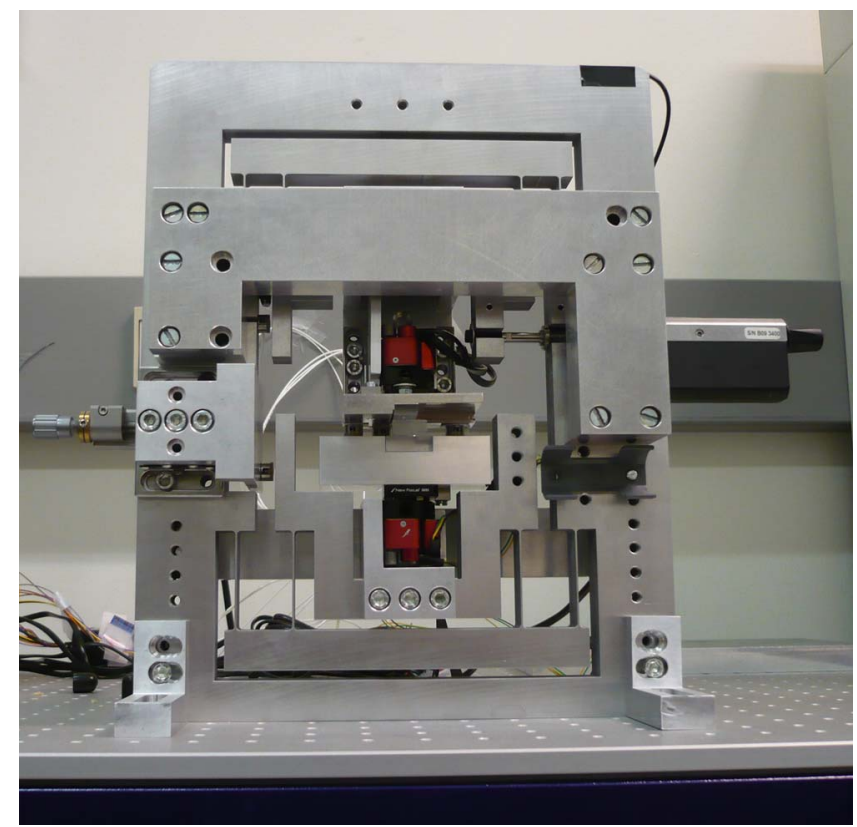

FIG. 3. X-FMR setup. The crossbar in front, that is not shown in the schematic Fig. 2, is added to stiffen the assembly and suppresses bending of the frame during operation.

plate of aluminum of $2 \mathrm{~cm}$ thickness. Eight regions of the plate have been machined down to narrow leaves (indicated as "springs" in Figure 2), which act as springs and hinges at the same time. Four such leave springs (connecting, for example, the "driven fixture" in the upper part of Figure 2 to the outer frame) combine to one compound flexure system that allows linear, spring-loaded motion. In such a compound flexure, the corresponding outer pair of leaves mirrors the flexure of an inner pair of leaves; it is this symmetry that allows displacements of the moving part on the order of millimeter, while orthogonal deviations remain on the nanometer scale. The general operating principle of the compound flexure and flexure-based translation has been described in detail elsewhere. ${ }^{41,49}$ For optimal similarity of all springs, the frame was cut from a single monolithic block of aluminum with wire electrical discharge machining (wire-EDM).

A fixture holding the upper shearing surface is mounted onto the upper flexure compound and is driven by a motor. The lower shearing surface is held by a lower fixture that connects to the lower compound flexure, whose displacement is proportional to the shear stress acting on the lower shearing surface via the sample that is confined in the micrometer gap between the surfaces. Inductive, non-contact distance sensors (SMT 9700-20N, KAMAN Instrumentation, Colorado Springs, $\mathrm{CO}$ ) monitor the position and displacements of both the upper and lower compound flexures and fixtures. The displacement of the upper fixture with the motor imposes a controllable strain onto the sample; the X-FMR is thus a strain-controlled rheometer. The displacement is typically performed in a saw-tooth fashion by moving the upper plate back and forth at constant velocities, thus subjecting the sample to a steady shearing deformation within a single deformation cycle. The motor is a DC servo-motor (LTA-HS, Newport, Irvine, CA) with a maximum velocity of $v_{\max }=5 \mathrm{~mm} / \mathrm{s}$ and a minimum incremental step of $100 \mathrm{~nm}$. Assuming that 10 steps/s can be considered a continuous rate, the minimum velocity is $v_{\min }=1 \mu \mathrm{m} / \mathrm{s}$. Together with the gap distance $h$, this determines the available shear rates $\dot{\gamma}=v / h$. Since $h$ can be set in the range 10-400 $\mu \mathrm{m}$, shear rates over a range of $10^{-3}-10^{3} \mathrm{~s}^{-1}$ can be applied. For rheological measurements in combination with the scattering experiments discussed in this paper, a lower limit for the gap of $10 \mu \mathrm{m}$ arises from the minimum diameter to which the X-ray beam could be focused in order to avoid parasitic scattering off the shear cell walls. Furthermore, entrapped airborne dust particles may dominate the stress response below gaps of about $3 \mu \mathrm{m} .{ }^{43,50,51}$ The available deformations are determined by the minimum incremental motion $\Delta x$ of the motor via $\gamma_{\min }=\Delta x / h$, as well as the gap and maximum travel distance $x_{\max }$ during a deformation cycle via $\gamma_{\max }=x_{\max } / h$. With $\Delta x_{\min }=100 \mathrm{~nm}$ and a maximum displacement of $x_{\max }=2 \mathrm{~mm}$, this yields strains of $2 \times 10^{-4}-2 \times 10^{2}(0.02 \%-20000 \%)$ that can be achieved for the above mentioned gap range.

The spring constant of the flexure systems was designed so that a deflection of $1 \mathrm{~mm}$ requires a force of $7.9 \mathrm{~N}$. The proximity sensors (SMT 9700-20 N, KAMAN Instrumentation, Colorado Springs, CO) have a range of $2 \mathrm{~mm}$ (full width) and accuracy of $15 \mathrm{~nm}$. Therefore, forces in the range $0.95 \mathrm{mN}-9.5 \mathrm{~N}$ can, in principle, be detected. However, it is advantageous for alignment purposes (see below) to limit the displacement of the detection fixture to about $50 \mu \mathrm{m}$, so that $0.12 \mathrm{mN}-60 \mathrm{mN}$ are measurable in practice. Using a shear cell with an area of $1 \mathrm{~cm}^{2}$, shear stresses $\sigma$ in the range 1.2$600 \mathrm{~Pa}$ are detectable. An example of stress measurements with the X-FMR exploring the accessible range at a typical gap setting of $h=20 \mu \mathrm{m}$ is given in Fig. 4(a) using Newtonian calibration oils of low and high viscosities of $1 \mathrm{Pas}$ and $60 \mathrm{~Pa}$ s, respectively.

The viscosities the X-FMR may measure are calculated by $\eta=\sigma / \dot{\gamma}$. Using the above mentioned stress and rate ranges gives a theoretical viscosity range of $1.2 \mathrm{mPas}-6$ $\times 10^{5} \mathrm{~Pa}$. Evidently, the design parameters limit the sensitivity to moderately and highly viscous samples, but in this way the setup is immune to vibrational noise, which can be difficult to control in an X-ray beamline environment. Figure 4(b) gives examples of viscosities measured at stresses above the indicated minimum stress level of $1.2 \mathrm{~Pa}$. One of these examples includes a latex suspension, composed of
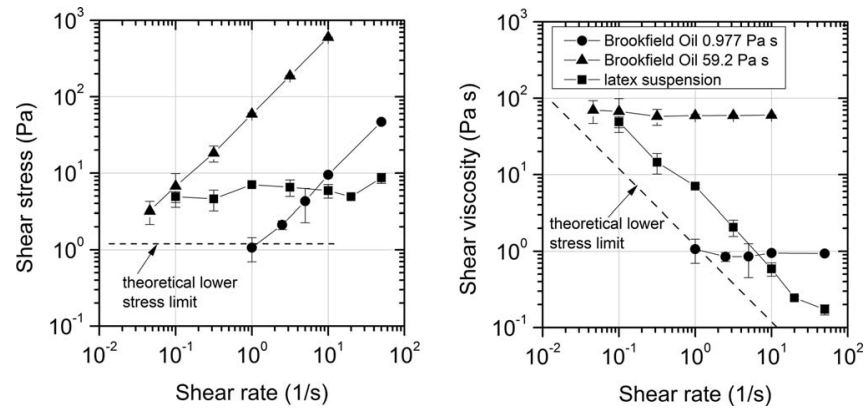

FIG. 4. Flow and viscosity curves determined with the X-FMR setup at a gap setting of $h=20 \mu \mathrm{m}$ for two calibration oils of nominal viscosities of 0.98 and 59.2 $\mathrm{Pa}$ s, as well as for the latex suspension further investigated in Fig. 9. 
poly(butylacrylate-styrene) spheres of $112 \mathrm{~nm}$ diameter and an effective volume fraction of 0.51 . Results on this sample will be discussed below, along with X-ray results on the same sample.

It should be noted that the stresses and viscosities reported in Fig. 4 are steady state values. Although the sliding plate rheometer is intrinsically limited in the deformation that can be applied during a single cycle in the saw-tooth deformation pattern, the small gap $h$ in combination with the relatively large travel distance $x$ allow for large deformations $\gamma_{\max }=x_{\max } / h$. For all measurements reported in this paper it was confirmed that the evolving stresses reached steady state within each individual deformation cycle.

\section{B. Shear cell}

Figure 5 shows a schematic drawing of the details of the sliding plate geometry and its assembly. The sample is sheared between two mirror substrate glasses (fused silica, flatness $\lambda / 10 \approx 60 \mathrm{~nm}$, Part \#048-0195, OptoSigma, Santa Ana, CA), which have been ground to the desired shape. The gap distance and relative orientation of the glass surfaces is monitored with three inductive distance sensors connected to the upper part, which sense the distance to the aluminum surface on the lower fixture. The sensors (SMT 9700-20 N, KAMAN Instrumentation, Colorado Springs, CO) have a range of $500 \mu \mathrm{m}$ and accuracy of $5 \mathrm{~nm}$.

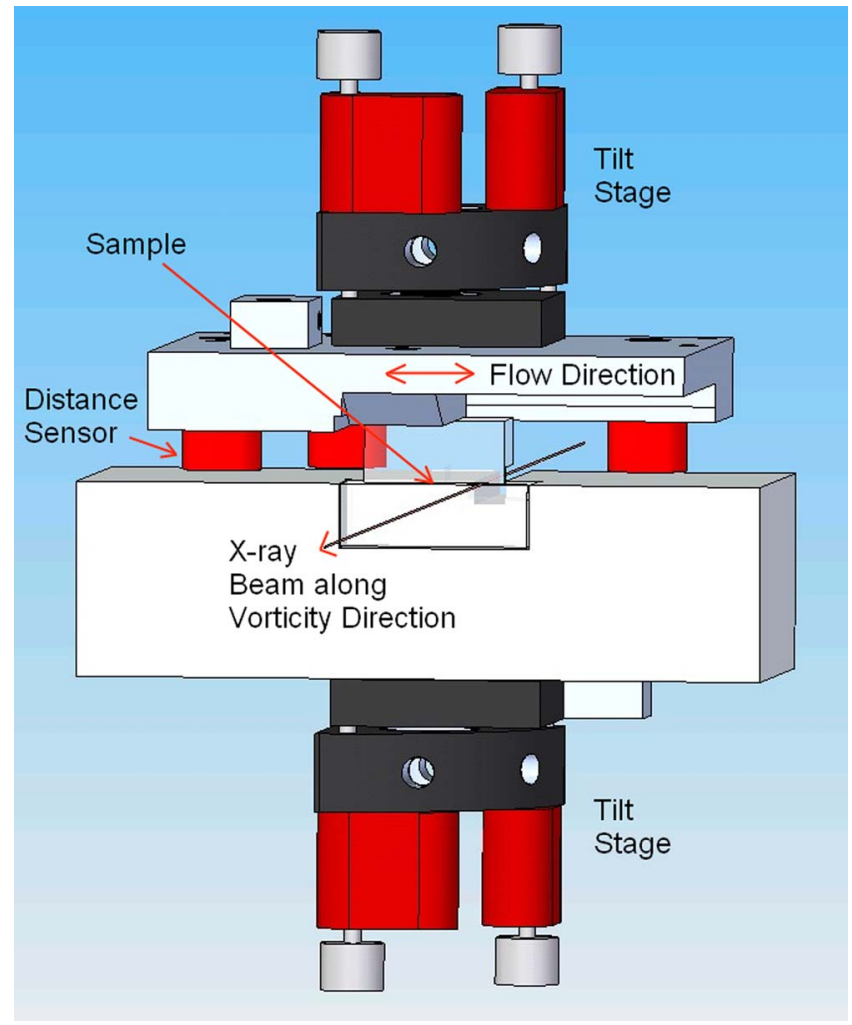

FIG. 5. CAD drawing of the sliding plate flow cell assembly. The sample is sheared between two mirror flat substrate glasses. The gap distance and relative orientation of the glass surfaces is monitored with three non-contact distance sensors on the upper part, which sense the aluminum surface on the lower part (width of the lower aluminum block for scale: $7.5 \mathrm{~cm}$ ). The tilt stages facilitate alignment of the shearing surfaces with respect to each other and the direction of travel (flow direction).
Both the upper and lower part of the cell assembly are mounted onto two-dimensional tilt stages with angular resolution $<1.5 \times 10^{-6} \mathrm{rad}$ (Model 8885, New Focus, San Jose, CA). This allows alignment of the shearing surfaces with respect to each other, as well as the direction of travel of the upper part. The lower tilt stage is attached directly to the lower compound flexure system. The upper tilt stage is mounted to a vertical translation stage (not shown here; smallest increment $<30 \mathrm{~nm}$; Model 9067-X-P, New Focus, San Jose, CA) that controls the gap distance. The glass parts and the lower aluminum block can be removed for easy cleaning.

The glass components that make up the flow cell are shown in Fig. 6. The upper glass is displaced horizontally with respect to the lower one, producing a homogenous linear shear flow field. The beam penetrates the sample in the vorticity direction, perpendicular to the $\mathbf{v}-\nabla \mathbf{v}$ plane. The scattering pattern obtained is a projection of the product of structure and form factors in the $\mathbf{v}-\nabla \mathbf{v}$ plane. To limit the beam path length, the cell has been designed to have a narrow part, which can be manufactured to thicknesses from several millimeters down to $0.5 \mathrm{~mm}$. In the experiments presented here, the thickness was on the order of $2 \mathrm{~mm}$, which is near the absorption length of our samples and thus optimizes the scattering signal strength. ${ }^{52}$ On the right side, the measurement geometry has a larger area of roughly $10 \times 10 \mathrm{~mm}^{2}$ to ensure sufficient area to have a measurable force signal. The dimensions of the measurement area can be changed to accommodate samples of different viscosities. Samples are typically loaded so that they cover the entire lower surface of the upper glass in all working positions. The sample-air interfaces penetrated by the beam are made vertical and reduced to the cell geometry

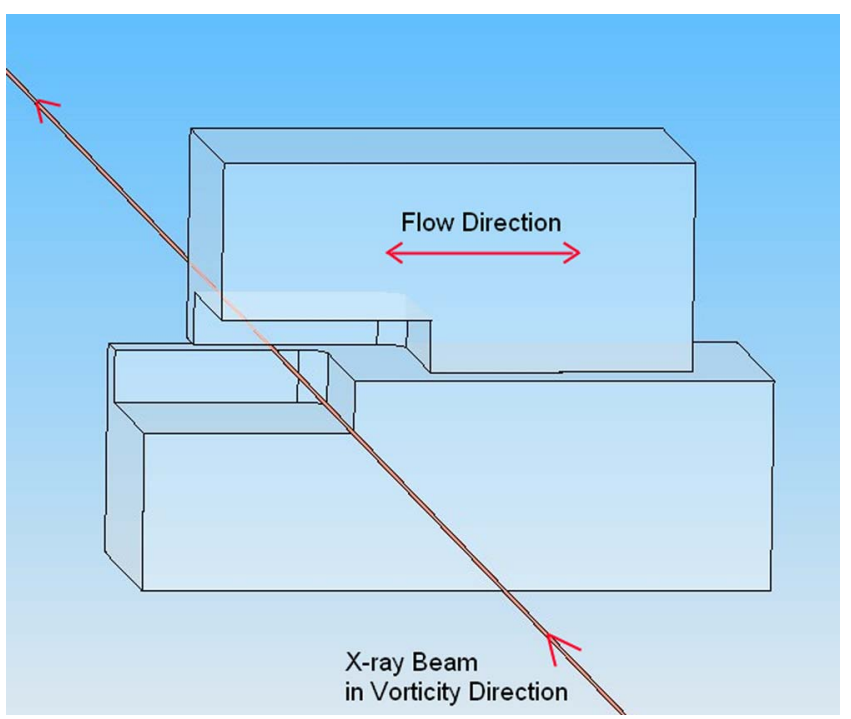

FIG. 6. Shear cell geometry, following the X-ray beam path. The upper glass is displaced with respect to the lower one, producing a plane Couette flow (width of the lower glass block for scale: $2.0 \mathrm{~cm}$ ). The beam penetrates the sample perpendicular to the $\mathbf{v}-\nabla \mathbf{v}$ plane (or in the vorticity direction, see Fig. 1). On the left hand side, the cell has a narrow part of $2 \mathrm{~mm}$ thickness for optimal scattering results. On the right hand side, it has a larger area of roughly $10 \times 10 \mathrm{~mm}^{2}$ to ensure a sufficient strong stress signal. The sample is loaded so that it covers the entire lower surface of the upper glass in all operating positions. The sample-air interfaces penetrated by the beam are made vertical and reduced to the cell geometry by scraping and effects of surface tension. 
by scraping. As the gap sizes are smaller than the capillary length scale, this further aides in obtaining relatively smooth interfaces. With an area of about $1 \mathrm{~cm}^{2}$, and at a gap distance of $100 \mu \mathrm{m}$ (for example), a sample volume on the order of $10 \mu \mathrm{l}$ suffices to fully fill the gap.

\section{Alignment procedures and sample loading}

The procedures used here are similar to those proposed by Baik et al. ${ }^{42}$ on a larger version of the FMR. First, the upper section of the shear cell assembly is loosened from the tilt stage and pressed onto the lower one by its own weight (approximately $300 \mathrm{~g}$ ). This is a stable and well-defined position since by the center of mass of the upper part is designed to coincide with the areal center of the shear cell. At this zerogap position, the readings of the three distance sensors are recorded and then the upper section of the shear cell is reattached to the tilt stage. Parallelism can now be produced to within $10^{-5} \mathrm{rad}$ at any gap distance, using the distance sensors and tilt stages.

In a next step, the misalignment of the plates with respect to the direction of travel of the upper driven fixture is minimized. This is achieved by moving the upper fixture at a gap between the surfaces of $\sim 100 \mu \mathrm{m}$ over distances on the order of $\mathrm{mm}$, while observing the distance sensor readings. The lower assembly is then tilted until parallelism of the lower glass surface with the upper travelling stage is optimal. The upper plate is then re-adjusted parallel to the lower one. Deviations amount to few $\mathrm{nm}$, thus obtaining parallelism better than $10^{-5} \mathrm{rad}$. Misalignment of the direction of travel of the lower fixture is negligible, since the typical stress-induced deflection is kept on the micron scale. Finally, the entire frame is mounted onto a xyz translation stage, and vertically displaced until the X-ray beam passes through the center of the cell, and tilted to align the X-ray beam parallel to the shearing surfaces.

Samples are loaded using a syringe. The upper cell wall is lifted manually while the sample is injected. In the present experiments, only the scattering region of the cell was loaded to reduce sample consumption; when fully loading the cell stress data are simultaneously recorded during SAXS. Following closure of the cell, the sample-air interfaces penetrated by the X-ray beam are made vertical and reduced to the cell geometry by scraping.

\section{EXPERIMENT}

The X-FMR has been tested on the microfocus SAXS beamline ID10A of the European Synchrotron Radiation Facility (ESRF), Grenoble, France. The beam was focused down to a cross section of about $10 \times 10 \mu \mathrm{m}^{2}$. The beam energy was set to $8.1 \mathrm{keV}$. The distance between the scattering center and the CCD camera was $1.2 \mathrm{~m}$. The CCD chip had 1277 $\times 1152$ pixel and dimensions of $28.7 \times 25.9 \mathrm{~mm}^{2}$. The CCD dimensions and position as well as the size of the utilized beam stop set the observable range of scattering vectors of $0.0065-0.045 \AA^{-1}$, corresponding to real space length scales of about $10-90 \mathrm{~nm}$. The exposure time was $1 \mathrm{~s}$ and thus within a single deformation cycle and at a constant rate for all shear experiments reported here. Shear experiments were repeated over several deformation cycles to assure reproducibility and to monitor the steadiness of the material functions to determine the onset of drying at the free sample surfaces or to detect other possible transient changes in the sample over several deformation cycles. For the water based systems investigated in this study, the presented data were, however, unaffected by sample drying and represent steady state values over several deformation cycles.

\section{A. Tilting of the nematic order in $f d$-virus suspensions}

The first case study is a suspension of the bacteriophage $f d$ virus, which is a monodisperse semi-flexible rod-like particle with a length of $880 \mathrm{~nm}$ and a bare particle diameter of $6.6 \mathrm{~nm} .{ }^{53}$ The $f d$ virus consist of a protein capsid encompassing a circular single strain DNA. Suspensions of these viruses in aqueous media are valuable model systems for suspensions of rod-like particles in general, due to their high length-todiameter (aspect) ratio, excellent monodispersity, and welldefined and controllable surface properties. ${ }^{54}$ The virus used here is termed $f d$-PEG since it is grafted with poly(ethylene glycol) of a molecular weight of $5 \mathrm{~kg} / \mathrm{mol}$ to impart steric stability. ${ }^{55}$ This surface modification results in approximately hard-core type interaction among the rods, similar to sterically stabilized spheres in bulk suspensions. ${ }^{56}$ The virus particles are suspended in a $110 \mathrm{mM}$ Tris buffer solution, to screen the electrostatic interactions. At high concentrations, the $f d$ virus forms lyotropic liquid crystalline phases mainly driven by the competition of orientational vs. positional entropy. ${ }^{57}$ Nematic $f d$ virus suspensions subjected to flow in bulk undergo a transition from director tumbling, wagging to flow-aligning. ${ }^{58} \mathrm{~A}$ key observable, which provides valuable insights into the nature of the nematic and is a critical test for constitutive models, is the tilt or Leslie angle. For flow-aligning nematics, the tilt angle of the director with the flow direction has a fixed value, independent of shear rate, as, for example, has been observed for SDS/Decanol nematics using flow-SANS in the $\mathbf{v}$ $\nabla \mathbf{v}$ plane. ${ }^{59}$ For tumbling nematics, the steady state, domain averaged orientation angle starts of at a small value $\left(1^{\circ}-2^{\circ}\right)$ and will tend to zero as the shear rate is increased. ${ }^{31,59}$

For the $f d$-suspension under investigation, the behavior is expected to be of the tumbling type in the accessible shear rate range. ${ }^{58}$ The X-FMR can now be used to detect the influence of flow and confinement on the nematic order. The concentration of the virus suspension was set to $68 \mathrm{mg} / \mathrm{ml}$ and thus well in the nematic domain. ${ }^{57}$ Due to possible solvent evaporation during the experiment, the actual averaged concentration may be slightly higher. Due to the structure of the virus, which is a single-strand-DNA filled protein capsid, the scattering contrast is not very high and, combined with the small beam size, the SAXS pattern not very intense. Even so, scattering patterns clearly show effects during shearing. An example of the effect of shearing is shown in Fig. 7. The presence of the broad peak off-center shows that a recurring length scale exists, in this case near $20 \mathrm{~nm}$. Given the dimensions of the particles ${ }^{55}$ (effective rod diameter $=12.6 \mathrm{~nm}$ ), the peaks correspond to the first order of the structure factor and thus the interparticle (core-to-core) distance, from which the 

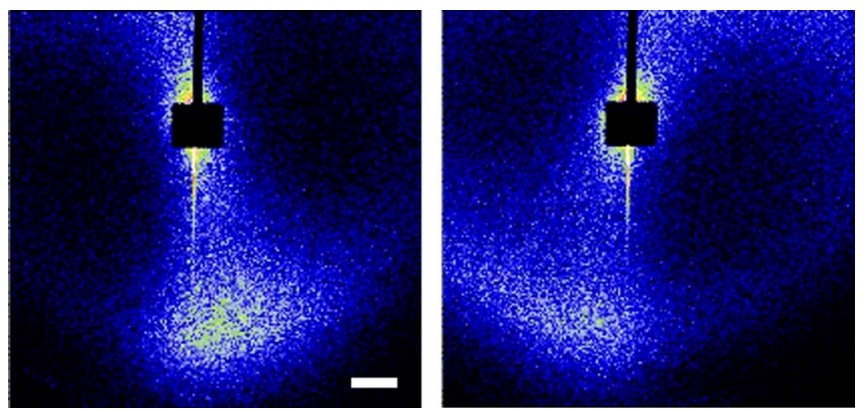

FIG. 7. Scattering patterns of $f d$-PEG virus, which approximates hard rods, suspended in $110 \mathrm{mM}$ Tris buffer solution and at a concentration of $68 \mathrm{mg} / \mathrm{ml}$. The bow-tie pattern indicates alignment of the rods, nearly parallel to the cell walls. The tilt of the preferred direction results from the shear flow; when the flow direction reverses, so does the tilt. This is visible only by probing the vorticity direction. Shear conditions: Gap distance: $20 \mu \mathrm{m}$; shear rate: $\pm 10 \mathrm{~s}^{-1}$. Scale bar: $0.1 \mathrm{~nm}^{-1}$.

distance between the side-by-side spaced rods can be concluded to be near $7 \mathrm{~nm}$. Since the peak is near the vertical axis in the pattern, the side-by-side arrangement is in the flow direction, at least on average. In principle, further ordering in the long direction of the particles could introduce higher order peaks, and this would modulate the lobe feature into a multi-peak structure.

More importantly, the nematic $f d$-systems serve to demonstrate the usefulness of the X-FMR as we will focus on the tilt of the patterns with respect to the flow direction. When the flow direction is reversed, the direction of tilting is reversed. It can be concluded that the X-FMR allows one to successfully investigate the orientation of the structure projected in the $\mathbf{v}-\nabla \mathbf{v}$ plane even at a gap of only $20 \mu \mathrm{m}$ (about 25 times the rod length). It should be noted that other configurations for flow-scattering, where the radiation is sent along the flow direction or the velocity gradient direction do not enable one to determine the orientation angle.

At a shear rate of $50 \mathrm{~s}^{-1}$, the tilting in this $f d$-PEG sample was so consistent that we could extract accurate values for the tilt angle under flow and confinement, as follows. In each image, the background was subtracted, and traces of the direct beam, parasitic scattering, and the beam stop were masked. Due to the small beam size the latter requires more attention as for bulk measurements with normal SAXS setups. To aid the intensity fitting procedure, the signal was subtracted by an average "isotropic background" value determined in the dark region inside the ring and away from the lobe. A robust procedure to obtain the instantaneous average orientation angle was obtained using the fit of a polar Gaussian intensity profile to each image

$$
I(q, \phi)=I_{\max } \exp \left[-\frac{\left(q-q_{\max }\right)^{2}}{2 \sigma_{q}^{2}}-\frac{\left(\phi-\phi_{\max }\right)^{2}}{2 \sigma_{\phi}^{2}}\right],
$$

where $q$ is the magnitude of the scattering vector, $\phi$ its azimuth, $I_{\max }$ the maximum intensity, $q_{\max }$ and $\phi_{\max }$ the position of the maximum, and $\sigma_{q}$ and $\sigma_{\phi}$ the dimensions of the lobe. $q=0$ corresponds to the beam center, determined by scattering from a hexagonal crystal (described in Sec. III B). $\phi=0$ corresponds to the negative vertical. The angle $\phi_{\max }$ obtained from the fitting is denoted the tilt angle (the subscript will be

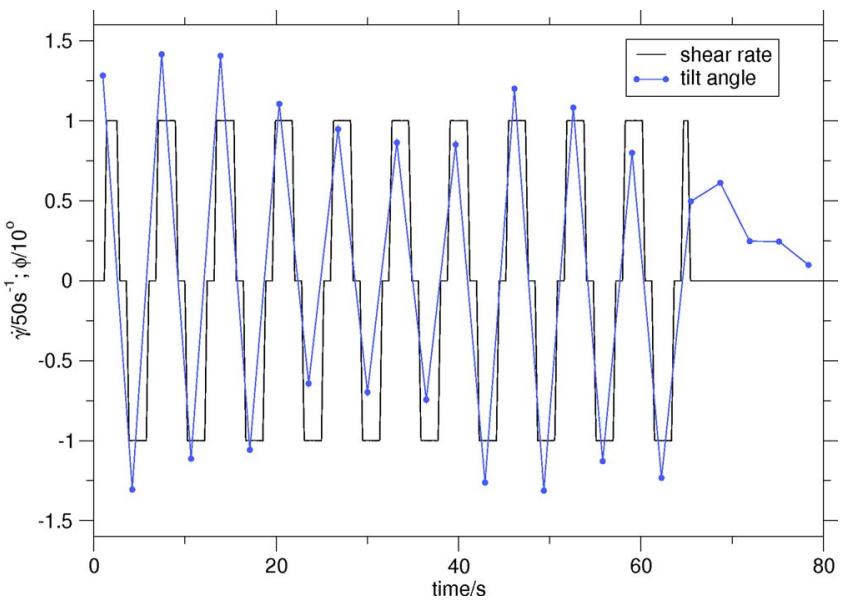

FIG. 8. Simultaneous shear and scattering experiment for the $f d$ virus solution confined in a gap of $20 \mu \mathrm{m}$. Shear rates and tilt angles (lines to guide the eye) as found by fitting polar Gaussians to the X-ray scattering images (see text for details). The tilt angle follows the shear rate, which alternates between $\pm 50 \mathrm{~s}^{-1}$. The preferred direction of rod orientation is, on average, deflected by $10.6^{\circ} \pm 0.5^{\circ}$.

omitted in the following). A tilt angle of $\phi=0$ refers to the nematic parallel to the flow direction. To quantify the correlation of the tilt angle to the shear rate, the tilt angle data were normalized by use of mean and standard deviation (rejecting not trustworthy data points at the beginning and end of the run), and the shear rate data were normalized to range from -1 to 1 . This normalized pair of data were then correlated, and if returning a value close to unity the tilt angle was concluded to follow the shear rate, and an average magnitude of tilt angle was calculated.

The shear rate and tilt angle data of the mentioned $f d$ PEG sample are shown in Fig. 8. The correlation evaluated to $0.973 \pm 0.004$ (statistical error), and the magnitude of the tilt angle was measured to be, on average, $10.6^{\circ} \pm 0.5^{\circ}$. The error of the latter is $\sigma / N^{-1 / 2}$, where $\sigma=2.3^{\circ}$ is the standard deviation and $N=19$ the number of included data points. Other sources of error, such as uncertainty in temporal synchronization and the influence of how many data points are excluded at beginning and end, were found to be negligible. Upon cessation of flow the orientation relaxed and became parallel to the confining walls. It can be concluded that the X-FMR setup is able to quantitatively measure structural evolution in tunable flow and confinement conditions.

\section{B. Lattice distortions of a colloidal crystal}

As a second test case, a suspension of sterically stabilized latex spheres was investigated. The colloidal particles are composed of a poly(butylacrylate-styrene) core and a shell of stabilizing surfactant. The sample used here is the same as the one labeled A1 in earlier studies. ${ }^{4,60,61}$ Steric repulsion dominates the particle interaction due to an adsorbed layer with a hydrodynamic thickness of $10 \mathrm{~nm} .{ }^{60}$ Hence, suspensions of such particles approximate hard-sphere behavior. The particle form factor was determined previously by the scattering from a dilute suspension. ${ }^{4}$ The average core diameter is $111.2 \pm 0.8 \mathrm{~nm}$. The stock suspension had an 
effective hard-sphere volume fraction of 0.62 as determined from rheological measurements. ${ }^{60,61}$ The sample was diluted in water to an effective volume fraction of 0.51 , which lies inside the solid-fluid coexistence region for hard spheres. ${ }^{56,62}$ However, due to possible solvent evaporation during the current experiment, this number again serves as a lower limit.

The rheological data (stress and viscosity) obtained at a gap setting of $h=20 \mu \mathrm{m}$ are given in Fig. 4. The sample exhibits the expected apparent yield stress behavior, with plastic flow at small Pe number. ${ }^{63}$ The range of shear rates and volume fraction correspond to the Strained Crystal regime in the nomenclature of the Chen-Ackerson-Zukoski description. However, it is the first time such systems can be investigated in the $\mathbf{v}-\nabla \mathbf{v}$ plane. Typical scattering patterns observed at this gap setting during shear flow at a shear rate of $10 \mathrm{~s}^{-1}$ are shown in Fig. 9. The scattering patterns are composed of smeared lines, modulated by peaks which is consistent with a rhcp packing of layers stacked parallel to the $\mathbf{v}-\boldsymbol{\omega}$ plane, ${ }^{63-65}$ but a detailed analysis of the scattering patterns lies beyond the scope of this work. The manner in which the close packed layers accommodate the flow is less clear. Stokesian dynamics simulation of non-Brownian, electrostatically stabilized particles have identified the mechanisms the close-packed layers undergo relative translation due to shear flow. ${ }^{66}$ The sequence of trajectories evolves from the zig-zag motion characteristic of the strained crystal to the rectilinear motion of the sliding layer mechanism. For the present case of the confined sterically stabilized systems, the measurements in the XFMR enable one to investigate the mechanism by providing experimental access to the average deformation of the crystal lattice under shear flow. The discussion here is limited to the inner diffraction nodes because form factor effects attenuate the higher-order reflections. The first-order peaks are masked by the beam stop. In Fig. 9(a), the upper shearing surface moves in the positive $x$-direction (to the right). The black dots highlight the diffraction peak positions. In Fig. 9(b), the direction of shear is reversed. The original peak positions of Fig. 9(a) are again shown as black dots, to indicate the displacement of the peaks. The arrows (not to scale) emphasize the displacement of the peaks as the shearing direction is

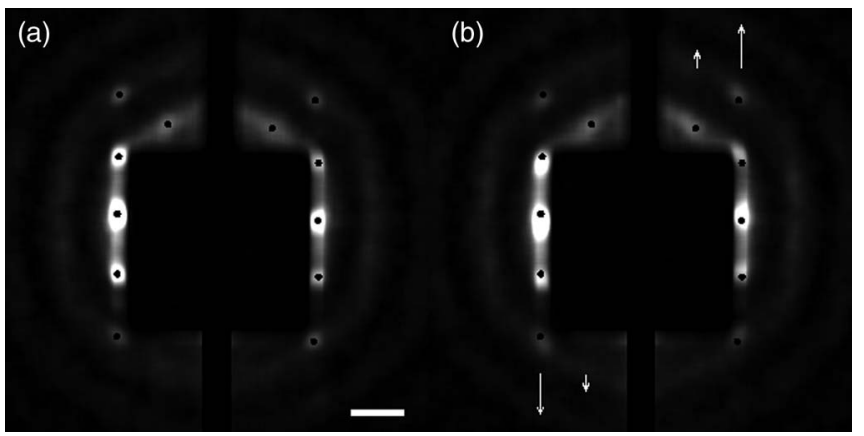

FIG. 9. Scattering patterns of a sterically stabilized poly(butylacrylatestyrene) latex sphere suspension under flow in a gap of $20 \mu \mathrm{m}$ at a shear rate of $10 \mathrm{~s}^{-1}$, observed in the vorticity direction of the flow field. (a) Upper shearing surface moves in the positive $x$-direction (to the right). The black dots highlight the peak positions. (b) Upper shearing surface moves in the negative $x$-direction (to the left). The black dots mark the original peak positions in Fig. 9(a). The arrows (not to scale) emphasize the displacement of the peaks as the shearing direction is reversed. Scale bar: $0.03 \mathrm{~nm}^{-1}$ reversed: Peaks to the left of the vertical symmetry axis are displaced downward, while peaks to the right are displaced upward. The farther away the peaks are from the vertical symmetry axis, the larger this displacement. This displacement of peaks is precisely the behavior expected for lattice distortions that follow the flow field. One can show that given a lattice deformation $\Delta x / y \equiv \gamma_{d}$ in real space, a deformation of $\Delta q_{y} / q_{x}=-\gamma_{d}$ results in $q$-space. Therefore, a distortion in the crystal lattice is manifest in the scattering pattern as an equally strong distortion rotated by $90^{\circ}$. Based on the shift of the peak positions and their separation from the vertical, we determined a magnitude of deformation of $\gamma_{d}=0.034(6)$ as a consequence of the shear flow. Note that this crystal lattice distortion can only be observed by probing the vorticity direction of the flow field. The scattering data are consistent with real space observations and 2D-FFT's by Stancik et al. ${ }^{67}$ on sheared 2D suspensions. The particles align into strings (in 2D) and planes (in 3D) of particles moving in the flow direction as this type of alignment allows slipping to occur with the least resistance. Contrary to the experiments on 2D suspensions by Stancik et al. ${ }^{67}$ a reorientation of the crystal lattice is not detected. This may be due to either the high volume fraction or the effect of confinement.

\section{CONCLUDING REMARKS}

A novel approach for combined scattering and rheology using a sliding plate rheometer has been proposed. A unique capability of the setup is the possibility to send the unaltered horizontal X-ray directly along the vorticity direction to simultaneously monitor the effects of confinement and shear flow on a complex fluid.

\section{ACKNOWLEDGMENTS}

We acknowledge the European Synchrotron Radiation Facility for provision of synchrotron radiation facilities and we would like to thank Anders Madsen and Orsolya Czakkel for assistance in using beamline ID10A. This work was supported by the FWO-Vlaanderen (Project Nos. G.0543.10 N and G.0697.11), and the European Union (EU) (Nanodirect Project Grant No. CP-FP 213948, ERC Starting Grant No. 203043-NANOFIB).

${ }^{1}$ F. Pignon, A. Magnin, J. M. Piau, B. Cabane, P. Lindner, and O. Diat, Phys. Rev. E 56, 3281 (1997).

${ }^{2}$ S. M. Jogun and C. F. Zukoski, J. Rheol. 43, 847 (1999).

${ }^{3}$ H. Versmold, S. Musa, C. Dux, P. Lindner, and V. Urban, Langmuir 17, $6812(2001)$

${ }^{4}$ P. Panine, T. Narayanan, J. Vermant, and J. Mewis, Phys. Rev. E 66, 022401 (2002).

${ }^{5}$ H. Hoekstra, J. Mewis, T. Narayanan, and J. Vermant, Langmuir 21, 11017 (2005).

${ }^{6}$ O. Diat, D. Roux, and F. Nallet, J. Phys. II 3, 1427 (1993).

${ }^{7}$ D. C. Roux, J. F. Berret, G. Porte, E. Peuvreldisdier, and P. Lindner, Macromolecules 28, 1681 (1995).

${ }^{8}$ F. R. Molino, J. F. Berret, G. Porte, O. Diat, and P. Lindner, Eur. Phys. J. B 3, 59 (1998).

${ }^{9}$ K. Hongladarom, V. M. Ugaz, D. K. Cinader, W. R. Burghardt, J. P. H. Quintana, B. S. Hsiao, M. D. Dadmun, W. A. Hamilton, and P. D. Butler, Macromolecules 29, 5346 (1996).

${ }^{10}$ W. R. Burghardt, Macromol. Chem. Phys. 199, 471 (1998). 
${ }^{11}$ H. Hoekstra, J. Vermant, J. Mewis, and T. Narayanan, Langmuir 18, 5695 (2002).

${ }^{12}$ D. K. Cinader and W. R. Burghardt, Polymer 40, 4169 (1999).

${ }^{13}$ G. Kumaraswamy, R. K. Verma, J. A. Kornfield, F. J. Yeh, and B. S. Hsiao, Macromolecules 37, 9005 (2004).

${ }^{14}$ G. Kumaraswamy, A. M. Issaian, and J. A. Kornfield, Macromolecules 32, 7537 (1999).

${ }^{15}$ P. K. Agarwal, R. H. Somani, W. Q. Weng, A. Mehta, L. Yang, S. F. Ran, L. Z. Liu, and B. S. Hsiao, Macromolecules 36, 5226 (2003).

${ }^{16}$ R. H. Somani, B. S. Hsiao, A. Nogales, S. Srinivas, A. H. Tsou, I. Sics, F. J. Balta-Calleja, and T. A. Ezquerra, Macromolecules 33, 9385 (2000).

${ }^{17}$ Y. P. Liu, W. Q. Zhou, K. P. Cui, N. Tian, X. Wang, L. B. Liu, L. B. Li, and Y. G. Zhou, Rev. Sci. Instrum. 82(4), 045104 (2011).

${ }^{18}$ S. Pujari, L. Dougherty, C. Mobuchon, P. J. Carreau, M. C. Heuzey, and W. R. Burghardt, Rheol. Acta 50, 3 (2011).

${ }^{19}$ L. M. C. Dykes, J. M. Torkelson, and W. R. Burghardt, Macromolecules 45, 1622 (2012).

${ }^{20}$ R. J. Plano, C. R. Safinya, E. B. Sirota, and L. J. Wenzel, Rev. Sci. Instrum. 64, 1309 (1993).

${ }^{21}$ J. A. Pople, I. W. Hamley, and G. P. Diakun, Rev. Sci. Instrum. 69, 3015 (1998).

${ }^{22}$ P. Panine, M. Gradzielski, and T. Narayanan, Rev. Sci. Instrum. 74, 2451 (2003).

${ }^{23}$ J. Vermant, P. Van Puyvelde, P. Moldenaers, J. Mewis, and G. G. Fuller, Langmuir 14, 1612 (1998).

${ }^{24}$ B. Struth, K. Hyun, E. Kats, T. Meins, M. Walther, M. Wilhelm, and G. Grubel, Langmuir 27, 2880 (2011).

${ }^{25}$ T. Meins, K. Hyun, N. Dingenouts, M. F. Ardakani, B. Struth, and M. Wilhelm, Macromolecules 45, 455 (2012).

${ }^{26}$ S. Kim, K. Hyun, Y. S. Kim, B. Struth, C. Clasen, and K. H. Ahn, Langmuir 29, 10059-10065 (2013).

${ }^{27}$ M. P. Lettinga, P. Holmqvist, P. Ballesta, S. Rogers, D. Kleshchanok, and B. Struth, Phys. Rev. Lett. 109, 246001 (2012).

${ }^{28}$ M. W. Liberatore, F. Nettesheim, N. J. Wagner, and L. Porcar, Phys. Rev. E 73, 020504(R) (2006).

${ }^{29}$ A. K. Gurnon, P. D. Godfrin, N. J. Wagner, A. P. R. Eberle, P. Butler, and L. Porcar, J. Vis. Exp. 84, e51068 (2014).

${ }^{30}$ M. E. Helgeson, L. Porcar, C. Lopez-Barron, and N. J. Wagner, Phys. Rev. Lett. 105, 084501 (2010).

${ }^{31}$ F. E. Caputo and W. R. Burghardt, Macromolecules 34, 6684 (2001).

${ }^{32}$ F. E. Caputo, V. M. Ugaz, W. R. Burghardt, and J. F. Berret, J. Rheol. 46, 927 (2002).

${ }^{33}$ J. L. S. Wales and H. Janeschitz-Kriegl, J. Polym. Sci. Part A-2: Polym. Phys. 5, 781 (1967).

${ }^{34}$ P. Baroni, C. Pujolle-Robic, and L. Noirez, Rev. Sci. Instrum. 72, 2686 (2001).

${ }^{35}$ F. E. Caputo, W. R. Burghardt, K. Krishnan, F. S. Bates, and T. P. Lodge, Phys. Rev. E 66, 041401 (2002).

${ }^{36}$ S. Pujari, S. Rahatekar, J. W. Gilman, K. K. Koziol, A. H. Windle, and W. R. Burghardt, J. Rheol. 55, 1033 (2011).
${ }^{37}$ A. J. Giacomin, T. Samurkas, and J. M. Dealy, Polym. Eng. Sci. 29, 499 (1989).

${ }^{38}$ S. G. Hatzikiriakos and J. M. Dealy, J. Rheol. 35, 497 (1991).

${ }^{39}$ N. R. Demarquette and J. M. Dealy, J. Rheol. 36, 1007 (1992).

${ }^{40}$ C. Clasen and G. H. McKinley, J. Non-Newtonian Fluid Mech. 124, 1 (2004).

${ }^{41}$ C. Clasen, B. P. Gearing, and G. H. McKinley, J. Rheol. 50, 883 (2006).

${ }^{42}$ S. J. Baik, P. Moldenaers, and C. Clasen, Rev. Sci. Instrum. 82, 035121 (2011).

${ }^{43}$ C. Clasen, H. P. Kavehpour, and G. H. McKinley, Appl. Rheol. 20, 196 (2010).

${ }^{44}$ C. Clasen, Rheol. Acta 51, 883 (2012).

${ }^{45}$ C. Clasen and G. H. McKinley, in Proceedings of the XIVth International Congress on Rheology, edited by J. W. Lee and S. J. Lee (The Korean Society of Rheology, 2004).

${ }^{46}$ N. Kojic, J. Bico, C. Clasen, and G. H. McKinley, J. Exp. Biol. 209, 4355 (2006).

${ }^{47}$ R. H. Ewoldt, C. Clasen, A. E. Hosoi, and G. H. McKinley, Soft Matter 3, 634 (2007).

${ }^{48}$ P. Erni, M. Varagnat, C. Clasen, J. Crest, and G. H. McKinley, Soft Matter 7, 10889 (2011).

${ }^{49}$ S. Gudlavalleti, B. R. Gearing, and L. Anand, Exp. Mech. 45, 412 (2005).

${ }^{50}$ A. Dhinojwala and S. Granick, J. Chem. Phys. 107, 8664 (1997).

${ }^{51}$ C. Clasen, J. Rheol. 57, 197 (2013).

${ }^{52}$ O. Glatter and O. Kratky, Small Angle X-ray Scattering (Academic Press, London, 1982).

${ }^{53}$ D. A. Marvin, Curr. Opin. Struct. Biol. 8, 150 (1998).

${ }^{54}$ Z. Dogic and S. Fraden, Philos. Trans. R. Soc. London, Ser. A 359, 997 (2001).

${ }^{55}$ E. Grelet and S. Fraden, Phys. Rev. Lett. 90, 198302 (2003).

${ }^{56}$ P. N. Pusey and W. Van Megen, Nature 320, 340 (1986).

${ }^{57}$ Z. Dogic and S. Fraden, Curr. Opin. Colloid Interface Sci. 11, 47 (2006).

${ }^{58}$ M. P. Lettinga, Z. Dogic, H. Wang, and J. Vermant, Langmuir 21, 8048 (2005).

${ }^{59}$ J. F. Berret, J. Vermant, and L. Noirez, in Proceedings of the 13th International Rheology Conference, 2000.

${ }^{60}$ L. Raynaud, B. Ernst, C. Verge, and J. Mewis, J. Colloid Interface Sci. 181, 11 (1996).

${ }^{61}$ J. Vermant, L. Raynaud, J. Mewis, B. Ernst, and G. G. Fuller, J. Colloid Interface Sci. 211, 221 (1999).

${ }^{62}$ W. G. Hoover and F. H. Ree, J. Chem. Phys. 49, 3609 (1968).

${ }^{63}$ L. B. Chen, B. J. Ackerson, and C. F. Zukoski, J. Rheol. 38, 193 (1994).

${ }^{64}$ J. Vermant and M. J. Solomon, J. Phys.: Condens. Matter 17, R187 (2005).

${ }^{65}$ I. P. Dolbnya, A. V. Petukhov, D. Aarts, G. J. Vroege, and H. N. W. Lekkerkerker, Europhys. Lett. 72, 962 (2005).

${ }^{66}$ J. J. Gray and R. T. Bonnecaze, J. Rheol. 42, 1121 (1998).

${ }^{67}$ E. J. Stancik, G. T. Gavranovic, M. J. O. Widenbrant, A. T. Laschitsch, J. Vermant, and G. G. Fuller, Faraday Discuss. 123, 145 (2003). 